\title{
Overview of material migration and mixing, fuel retention and cleaning of ITER-like castellated structures in TEXTOR
}

\author{
A. Litnovsky ${ }^{1}$, V. Philipps ${ }^{1}$, P. Wienhold ${ }^{1}$, A. Kreter $^{1}$, A. Kirschner ${ }^{1}$, D. Matveev ${ }^{1,2}$, \\ S. Brezinsek ${ }^{1}$, G. Sergienko ${ }^{1}$, A. Pospieszczyk ${ }^{1}$, B. Schweer ${ }^{1}$, C. Schulz ${ }^{1}$, O. Schmitz ${ }^{1}$, \\ J.W. Coenen ${ }^{1}$, U. Samm ${ }^{1}$, K. Krieger ${ }^{3}$, T. Hirai ${ }^{4}$, B. Emmoth ${ }^{5}$, M. Rubel ${ }^{5}$, B. Bazylev ${ }^{6}$, \\ U. Breuer ${ }^{7}$, A. Stärk ${ }^{7}$, S. Richter ${ }^{8}$, M. Komm ${ }^{9}$ \& TEXTOR Team.
}

${ }^{1}$ Institut für Energieforschung - Plasmaphysik, Forschungszentrum Jülich, Trilateral Euregio Cluster, Association EURATOM- FZ Jülich, D-52425 Jülich, Germany;

${ }^{2}$ Ghent University, Trilateral Euregio Cluster, Rozier B-9000, Ghent, Belgium;

${ }^{3}$ Max-Planck-Institut für Plasmaphysik, D-85748, Garching, Germany; ${ }^{4}$ ITER Organization, 13067 St Paul-lez-Durance, France;

${ }^{5}$ Alfvén Laboratory, KTH, Association EURATOM - VR, Stockholm, Sweden;

${ }^{6}$ Institut für Hochleistungsimpuls und Mikrowellentechnik, Karlsruher Institut für Technologie, 76021, Karlsruhe, Germany;

${ }^{7}$ Zentralabteilung für Chemische Analysen, Forschungszentrum Jülich, D-52425, Jülich, Germany;

${ }^{8}$ Gemeinschaftslabor für Elektronenmikroskopie, RWTH Aachen, D-52056, Aachen, Germany;

${ }^{9}$ Department of Surface and Plasma Science, Charles University, CZ-18000 Prague, Czech Republic.

\section{Abstract}

In ITER, plasma-facing components (PFCs) will be castellated by splitting them into small-size blocks to maintain the thermo-mechanical stability. However, there are still concerns, especially on the performance of castellated structures under high heat loads and on retention of radioactive fuel and 
impurities in the gaps. The R\&D program on ITER-like castellated structures is underway in TEXTOR addressing the physics and design issues of castellation.

Studies of material migration and fuel inventory are performed using dedicated exposures of the castellated limiters from high-Z materials in TEXTOR plasmas and long-term experiments using instrumented toroidal limiter tiles. Significant impurity transport to gaps of castellated limiters was detected: about $10 \%$ of impinging carbon flux originating from the graphite PFCs of TEXTOR was accumulated in the gaps. Results agree well with findings from the long-term exposures and can be quantitatively reproduced with 3DGAP code using the plasma background near the gap surfaces from particle-in-cell (PIC) code.

Intermixing of metals in the deposited layers provides difficulties for the gap cleaning. In recent experiments on TEXTOR, the deposits contained up to 70 at. \%. of tungsten (W) on the uppermost plasma-closest areas of gaps. Deposition in the gaps and metal mixing in the deposited layers demand for development of effective cleaning techniques for gaps. In experiments with ITERlike castellation, the gaps were successfully cleaned from carbonaceous deposits using oxygen plasmas at $350^{\circ} \mathrm{C}$. A contribution contains an overview of experimental and modeling results along with recommendations for plasma-facing components in ITER.

PACS: 52.55.Fa; 52.40.Hf; 52.25.Vy

JNM Keywords: Plasma-material interactions (P0500), Surface effects (S1300), Carbon (C0100), Divertor materials (D0500); Cladding materials (C0500);

PSI 18 Keywords: Deuterium inventory, Erosion and Deposition, High-Z limiter, Tungsten, TEXTOR Corresponding and presenting author: Dr. Andrey Litnovsky

Corresponding and presenting author address: Institut für Energieforschung - Plasmaphysik, Forschungszentrum Jülich, D-52425 Jülich, Germany Corresponding and presenting author E-mail: a.litnovsky@fz-juelich.de 


\section{Introduction}

Plasma-facing components in ITER will be exposed to intensive particle and radiation fluxes imposing the technological and engineering challenges to the design of PFCs. To alleviate the thermomechanical constraints and to ensure the durability of the plasma-facing components it was decided to split the entire first wall and divertor to a number of compact cells - to introduce the so-called castellated structures [1, 2]. However, an introduction of a castellation along with the significant reduction of the risk of the PFC destruction, has triggered a number of critical issues: the radioactive fuel may become accumulated in the gaps of castellated structures [4-6] and the power handling may degrade significantly due to hot spots introduced by the castellation.

While the aspects of power handling are the subject of the other paper [7], here we will focus our review on studies of the impurity deposition and fuel accumulation in the gaps of castellated structures based on experiments on TEXTOR and accompanying modeling and finally, on gap cleaning using hydrogen and oxygen plasmas.

\section{Erosion and deposition in the gaps of castellation structures}

\section{a. Discharge-resolved short-term experiments in TEXTOR}

To investigate the general nature of the impurity and fuel transport into the gaps under welldiagnosed plasma conditions, series of experiments were made in the scrape-off layer (SOL) plasmas of TEXTOR. The principal scheme of all the experiments was the same. The castellation made of TZM alloy (an alloy containing 99 at.\% of molybdenum) and machined to ITER relevant sizes of $10 \times 10 \times 10 \mathrm{~mm}$. Afterwards, the manufactured castellation was mounted on the roof-like test limiter 
and exposed in the SOL plasmas of TEXTOR for series of identical repetitive high performance discharges.

During the first experiment, the castellation was exposed to the total fluence of $4.9 * 10^{19}$ part. $/ \mathrm{cm}^{2}$ averaged over the area of a castellation with the surface temperature reaching $700^{\circ} \mathrm{C}$, as measured with a pyrometer on the uppermost part of the castellation. Visual inspection reveal the presence of the deposits both on the plasma-wetted top surface and in the gaps, therefore this experiment is referred to as a "deposition experiment" [8].

The second experiment was conducted under the conditions of net erosion: the plasma wetted top surfaces were metallically shiny, where in the gaps the deposition patterns were detected. The limiter was exposed for 39 plasma discharges, with the total flux averaged over an area of the castellation corresponds to $20^{*} 10^{20}$ part./ $\mathrm{cm}^{2}$ [8]. This is experiment is referred as "erosion" experiment.

Visual inspection of the gaps from both exposed limiters revealed plenty of experimental similarities. In the both experiments, the deposits in the gaps were detected on the very thin stripe-like zones in immediate vicinity of the plasma-closest edges of the gaps as illustrated on Fig.1. The efolding length of the carbon-containing deposit measured with several ion beam and electron beam surface diagnostics was around 1.0 - $1.8 \mathrm{~mm}$ for both experiments.

However, there were also the significant differences in the results of the experiments. The most important is the difference in the amount of impurities deposited on the plasma-wetted top surfaces and in the gaps. For the deposition experiment the most of impurities was found on the top surfaces: $\mathrm{C}_{\text {top }} / \mathrm{C}_{\text {gaps. }}$ 3 whereas for erosion experiment an amount of impurities on the top surfaces was essentially zero, while in the gaps the deposits with a thickness of up to $500 \mathrm{~nm}$ were detected. This finding outlines clearly the fact, that despite for the erosion-dominated conditions at the top surface, the screening and attenuation of the eroding particle fluxes was effective enough to turn these conditions to deposition-dominated inside the gaps. 


\section{b. Long-term exposure}

To investigate the impurity transport into the gaps on a long-term basis and to correlate these results with results of short-term exposure, two blades of the ALT II (Advanced limiter Test II) [9] were coated with a silicon layer having the thickness of 300-400 nm. This layer was supposed to serve as a marker layer, helping to evaluate erosion and deposition occurred at the blades. The special catcher plate was installed at the bottom of the gap formed by the two instrumented ALT II blades. The blades were then installed into TEXTOR and exposed for the entire campaign comprising 9500 plasma seconds, including 7 boronizations and accounting for total fluence of $2.9 * 10^{21}$ part./cm ${ }^{2}$ averaged over plasma-wetted top surface. Several surface analyses were applied to study the impurity deposition in the gaps and in particular, the deposition pattern. The deposits with a thickness up to 30 um were detected at the plasma-closest parts of gaps in a full similarity with the results of the short-term exposures, outlining the common nature of the physical processes governing the impurity transport into the gaps both for the short-term and long-term experiments. At the same time, rather thick deposits of up to $1 \mathrm{um}$ were firstly found at the bottom or exposed gaps. While there are several hypotheses to explain the observed deposition patterns however, the explanations remain to be of speculative nature and more investigations are certainly needed to understand the physical processes leading to the detected enhanced deposition at the bottom.

\section{c. Mitigation of deposition by the shaping of castellation}

Both the results of dedicated short-term experiments and the long-term exposure demonstrate, that the deposition in the gaps remains significant independently on whether erosion- or depositiondominated conditions are at the plasma-wetted surface of castellation. This fact calls for development of the deposition mitigation techniques. 
Optimization of the geometry of the cells of a castellation seems to be a natural way of mitigating the impurity transport into the gaps by eliminating the areas of gaps directly facing the plasma and contributing to the enhanced transport of the impurities into the gaps. Such areas are called "plasmaopen areas". To evaluate the effect of cell geometry on the mitigation of the impurity deposition in the gaps, the specially-designed double roof limiter was used in the recent experiment on TEXTOR. Castellation cells of two geometries were used: conventional, rectangular cells along with cells of a new roof-like geometry to shadow the plasma-open areas of the gaps from the plasma as it is illustrated on Fig. 2. The castellation of both geometries was exposed in a same experiment in the same plasma conditions allowing for the direct comparison between two exposed geometries of a castellation [10].

Investigations after the exposure have shown that a shaping of the castellated cells may indeed lead to the reduction of the impurity deposition and fuel accumulation. However, the decrease of carbon deposition was not very significant hardly exceeding $30 \%$ as compared with castellated structure with rectangular cells. In a similarity with the results from the long-term experiment, the deposits reaching $200 \mathrm{~nm}$ were detected at the bottom of the castellation. The measured deposition at the bottom corresponds to about $14 \%$ of the total impurity accumulation in the gap. These findings underline the fact, that the better understanding of physical processes leading to the impurity deposition in the gaps is required to gain the control over deposition. The significant intermixing of the sputtered tungsten into deposits at the upper part of the plasma-open areas of gaps was detected. The atomic fraction of tungsten reached 70 at.\%. Such a massive metal intermixing will provide the difficulties for removal of impurities from the gaps.

\section{d. Modeling of deposition in gaps}


Physical processes occurring inside the gap were modeled with the new Monte-Carlo code 3DGAP in the flexible 3D geometry. The detailed description of the code may be found in [11]. The following models were implemented into the code and used for evaluation of the deposition patterns:

- Particle reflection: cosine, isotropic or specular angular distribution

- Reflection coefficient: pre-defined or fitted from TRIM [12]

- Elastic neutral collisions with molecules of residual gas

- Plasma penetration into the gap (coupling with PIC simulations)

- Homogeneous surface mixing model for surface concentrations

- Simple model for chemical erosion

The parametric studies were made. The simulated data was matched with experimentally measured profiles of deposition. For simulations, the edge plasma parameters from either He-beam diagnostic or the probe diagnostic were used. The experimental data was afterwards matched with the particle-incell (PIC) code SPICE2 $[13,14]$ and translated inside the gap, where experimental measurements were impossible.

As it was inferred from the results of modeling, the elastic collisions of particles with the molecules of the residual gas inside the gap play a negligible role in the distribution of the impurities inside the gap. At the same time, an introduction of chemical erosion reaction chains into the code has proven to significantly change the distribution of carbon in the gap. Coupling with the tracing of particle reflection from the side walls of a gap and taking into account angular distributions of reflected particles, have provided better matching with experimental results and allowed for a quantitative agreement with measured deposition patterns illustrated in Fig. 3. Nevertheless, the deposition at the bottom of the gap still could not be reproduced by 3DGAP leaving the room for the further optimization of the modeling algorithms. Further details of modeling may be found in [15]. 


\section{d. Cleaning of the deposits inside the gaps}

Assuming the deposition in the gaps is yet to happen in ITER despite the mitigation techniques, the development of the effective gap cleaning is of significant importance. In an effort to address this issue, several plasma-discharge techniques were tested under the laboratory plasma conditions [16] in the “TOMAS” toroidal device [17]. The castellated cells of ITER-like geometry: $10 \times 10 \times 10 \mathrm{~mm}$ were pre-coated with an amorphous hydrocarbon film. The properties of this film were measured in the MirrorLab [18] at the Forschungszentrum Juelich. The pre-coated and pre-characterized castellated samples were then inserted into the glow- or electron-cyclotron resonance heated plasma and exposed in TOMAS. Hydrogen and oxygen plasmas were applied to evaluate the relative cleaning effectiveness. Cleaning cycles were carried out at temperatures of $200^{\circ} \mathrm{C}$ and $350^{\circ} \mathrm{C}$. The main results are provided in Fig. 4. As it can be seen from the figure, the most effective cleaning action can be achieved during the treatment in oxygen plasmas and the effectiveness of such a cleaning in the gaps is only marginally dependant on the temperature of the sample. The complete cleaning of the studied gaps was not achieved, unfortunately. However, the obtained results clearly demonstrate that the most successful cleaning was attained at the plasma-closest areas of the gaps and at the gaps bottom, i.e. at the locations where the most of the deposition and fuel accumulation is usually detected, accounting for up to $90 \%$ of the total inventory in the gaps.

\section{Summary and outlook}

A coherent research program is underway at TEXTOR tokamak to address the physics issues related to castellated structures for the ITER first wall and divertor. Impurity deposition and fuel accumulation in the gaps of castellated structures and possible ways to mitigate such deposition, castellation melting and melt layer motion, cleaning of the castellated structures are among the critical issues of the performance of castellation in ITER. The research program comprises the dedicated discharge- 
resolved short term experiments, long-term exposures of various castellated structures in TEXTOR, the dedicated modeling and laboratory experiments.

According to the results of experiments in TEXTOR, the deposition in the gaps of the castellated structures occurs independently on whether erosion- or deposition-dominated conditions prevail at the plasma-wetted surface of a castellation. This makes the gaps the giant reservoir for impurity and fuel accumulation in the future fusion devices, like ITER. The results of short-term experiments largely agree with the those of long-term exposures, demonstrating the common nature of the physics processes governing the deposition in the gaps in these experiments.

To gain the better understanding of these processes, the dedicated modeling effort is underway. The 3D Monte-Carlo 3DGAP code is used for the modeling, the plasma background at the vicinity of castellation is supplied from the experimental data and traced further inside the gaps using SPICE2 particle-in-cell code. Quantitative agreement of modeled and experimental patterns of carbon deposition in the gaps was reached. Particle reflections, including the angle distribution of reflected particles inside the gap coupled with chemical erosion, are playing the decisive role in the impurity deposition in the gaps. At the same time, the significant deposition at the bottom of gaps observed both in the short-term and long-term experiments at TEXTOR could not be reproduced by the modeling, manifesting the fact that some important physics may be still missing and calling for the further dedicated experiments and improvement of modeling algorithms.

The formation of mixed metal-containing layers in the gaps of castellated structures exposed under erosion conditions in TEXTOR will likely provide the severe difficulties in the gap cleaning. The significant effort is now focused on studies of cleaning of castellated structures using plasma discharges. The current studies show, that the highest efficiency of deposit removal is attained using the electron-cyclotron discharge in oxygen on the plasma-closest uppermost areas of the gaps and at the bottom of the castellation structures - in the areas where the maximum of deposition usually 
occurs in tokamak experiments. Whether it is possible to remove the metal-containing mixed layers formed during tokamak exposures remains to be studied.

The future investigations on gaining the better understanding of physical processes responsible for the impurity deposition in the gaps, mitigation of deposition, code improvement and further cleaning tests on ITER-relevant deposits originating from tokamak exposures.

\section{Acknowledgments}

The authors would like to thank the European and international laboratories: Max-Planck Institut für Plasmaphysik, Alfven Laboratory, Karlsruher Institut für Technologie and universities: Ghent University, Charles University in Prague and RWTH Aachen University for the very efficient help and friendly support in these studies. This work is being performed within the research program of the European Task Force on Plasma-Wall Interactions and is a part of the IEA-ITPA Joint Experiments Program, task DSOL 13. 


\section{References:}

1. A Review of ITER Technology R\&D, Fus. Eng. Des. (special issue) 55 (2-3) 2001, Chapters 3.3 and 3.4;

2. W. Daenner, M. Merola, P. Lorenzetto, A. Peacock, I. Bobin-Vastra, L. Briottet, P. Bucci, D. Conchon, A. Erskine, F. Escourbiac, M. Febvre, M. Grattarola, C.G. Hjorth, G. Hofmann, A. Ilzhoefer, K. Lill, A. Lind, J. Linke, W. Richards, E. Rigal, M. Roedig, F. Saint-Antonin, B. Schedler, J. Schlosser, S. Tahtinen, E. Visca, Fus. Eng. Des. 61\&62 (2002) 61;

3. K. Krieger, W. Jacob, D.L. Rudakov, R. Bastasz, G. Federici, A. Litnovsky, H. Maier, V. Rohde, G. Strohmayer, W.P. West, J. Whaley, C.P.C. Wong, J. Nucl. Mater. 363-365 (2007) 870;

4. D. Rudakov, W. Jacob, K. Krieger, A. Litnovsky, V. Philipps, W.P. West, C.P.C. Wong, S.L. Allen, R.J. Bastasz, J.A. Boedo, N.H. Brooks, R.L. Boivin, G. De Temmerman, M.E. Fenstermacher, M. Groth, E.M. Hollmann, C.J. Lasnier, A.G. McLean, R.A. Moyer, P.C. Stangeby, W.R. Wampler, J.G. Watkins, P. Wienhold, J. Whaley, Phys. Scr. T128 (2007) 29;

5. D.G. Whyte, J.P. Coad, P. Franzen, H. Maier, Nucl. Fusion 39 (1999) 1025;

6. M. Rubel, J.P. Coad, P. Wienhold, G. Matthews, V. Philipps, M. Stamp, T. Tanabe, Phys. Scr. T111 (2004) 112;

7. J.W. Coenen, B. Bazylev, S. Brezinsek, V. Phillips, T. Hirai, A. Kreter, G. Sergienko, A.Pospieszczyk, Y. Ueda, T. Tanabe, U. Samm and the TEXTOR-Team “Tungsten Melt Layer Motion on Castellated Surfaces at the Tokamak TEXTOR”, these proceedings;

8. A. Litnovsky et al. J. Nucl. Mater. 367-370 (2007) 1481;

9. K. H. Finken D. Reiter, T. Denner, K. H. Dippel, J. Hobirk, G. Mank, H. Kever, G. H. Wolf, N. Noda, A. Miyahara, T. Shoji, K. N. Sato, K. Akaishi, J. A. Boedo, J. N. Brooks, R. W. Conn, W. J. Corbett, R. P. Doerner, D. Goebel, D. S. Gray, D. L. Hillis, J. Hogan, R. T. McGrath, M. 
Matsunaga, R. Moyer, R. E. Nygren, J. Watkins, Fus. Sci. and Tech., vol. 47, No. 2 (2005), 126;

10. A. Litnovsky, P. Wienhold, V. Philipps, K. Krieger, A. Kirschner, D. Matveev, D. Borodin, G. Sergienko, O. Schmitz, A. Kreter, U. Samm, S. Richter, U. Breuer and TEXTOR Team, J. Nucl. Mater. 390-391 (2009) 556;

11. D. Matveev, A. Kirschner, A. Litnovsky, M. Komm, D. Borodin, V. Philipps and G. Van Oost „Modelling of impurity deposition in gaps of castellated surfaces with the 3DGap code“, accepted for publishing in Plasma Physics and Controlled Fusion;

12. W. Möller, Comput. Phys. Commun., 1988;

13. SPICE2 code (Sheath-Particle-in-Cell) http://spice2.sourceforge.net;

14. R. Dejarnac and J. Gunn, J. Nucl. Mater., vol. 363-365 (2007), 560-564;

15. D. Matveev, A. Kirschner, A. Litnovsky, A. Kreter, P. Wienhold, M. Komm, D. Borodin, and G. Van Oost "Modelling of impurity deposition in gaps of castellated structures in TEXTOR with the 3DGap code”, these proceedings;

16. A. Kreter, C. Schulz, V. Philipps, A. Litnovsky and U. Samm „Fuel removal from castellated structures by plasma discharges in reactive gases”, these proceedings;

17. H.B. Störk, J. Winter, J.Ihde, H.G. Esser, H. Reimer and M. Freisinger, Fus. Tech. vol. 39 No. 1 (2001) 54;

18. https://tec.ipp.kfa-juelich.de/mirrorlab/ Access details: mirrorlab@fz-juelich.de; 


\section{Figure captions:}

Figure 1. Carbon deposition in the gaps of the castellated limiter exposed in TEXTOR

Figure 2. A view of a castellated limiter with two shapes of castellation: rectangular-conventional and shaped - to mitigate the deposition and the scheme of exposure.

Figure 3. Comparison of modeled carbon deposition pattern (continuous line) with experimental results (squares). A constant chemical erosion yield $\mathrm{Y}=2.0 \%$ was used in the modeling.

Figure 4. Comparison of deposit removal efficiency, normalized to the impinging plasma flux at the plasma-wetted top surface of the castellation. The data shown for various discharges in oxygen and hydrogen and for various temperatures of the castellation during the cleaning. 


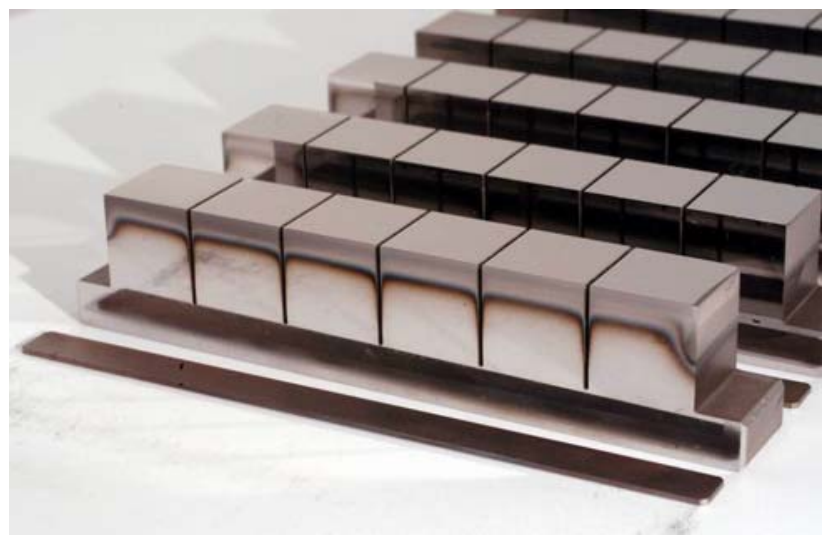

Figure 1. Carbon deposition in the gaps of the castellated limiter exposed in TEXTOR 


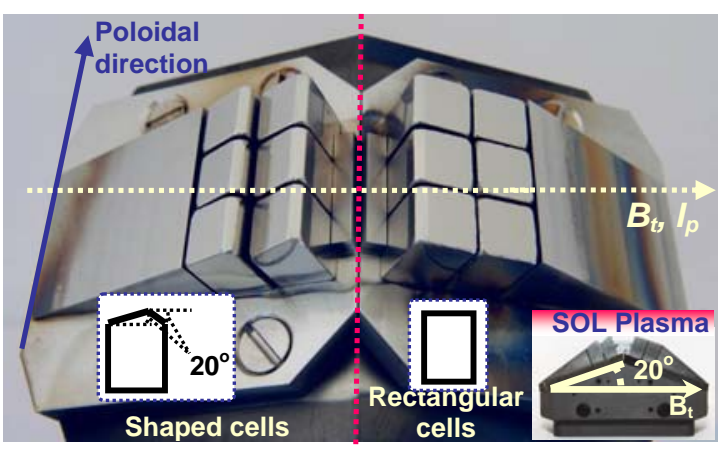

Figure 2. A view of a castellated limiter with two shapes of castellation: rectangular -conventional and shaped - to mitigate the deposition and the scheme of exposure. 


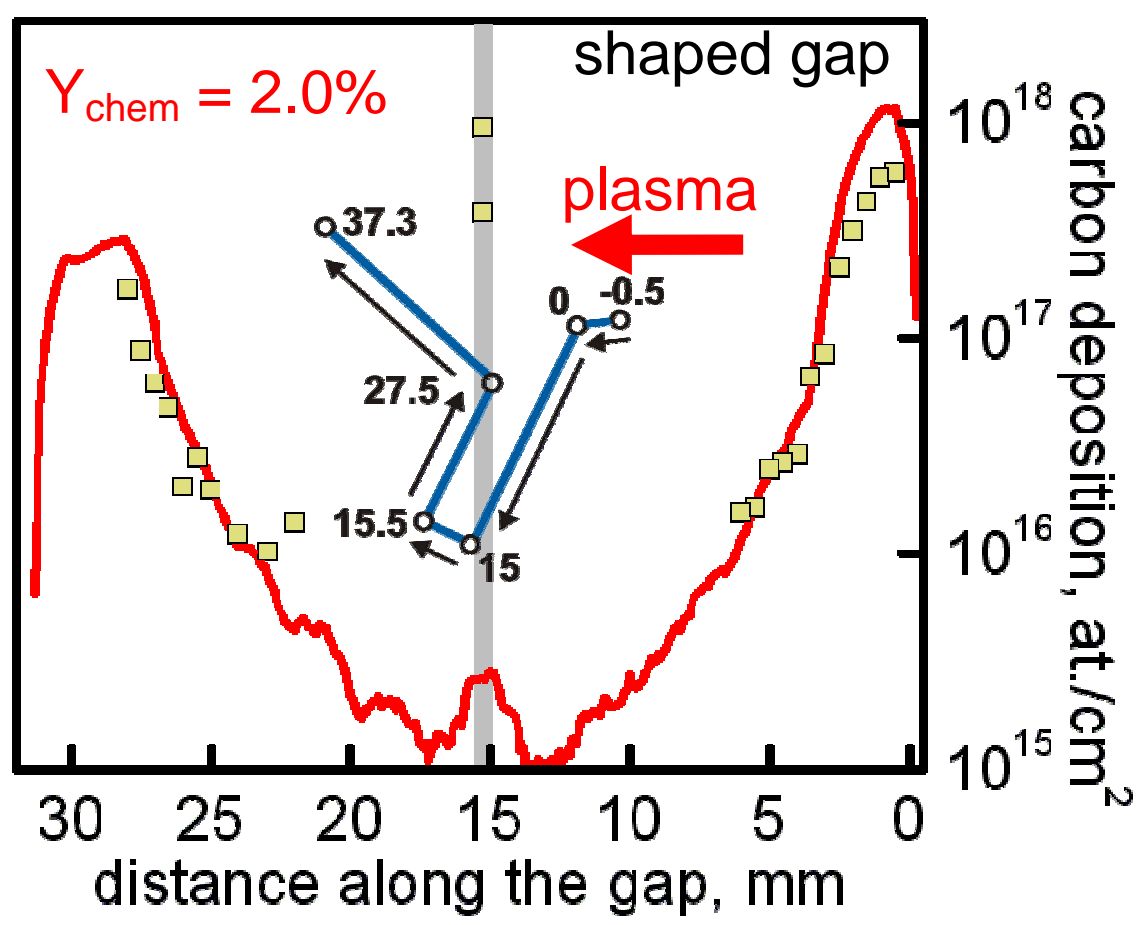

Figure 3. Comparison of modeled carbon deposition pattern (continuous line) with experimental results (squares). A constant chemical erosion yield $\mathrm{Y}=2.0 \%$ was used in the modeling. 


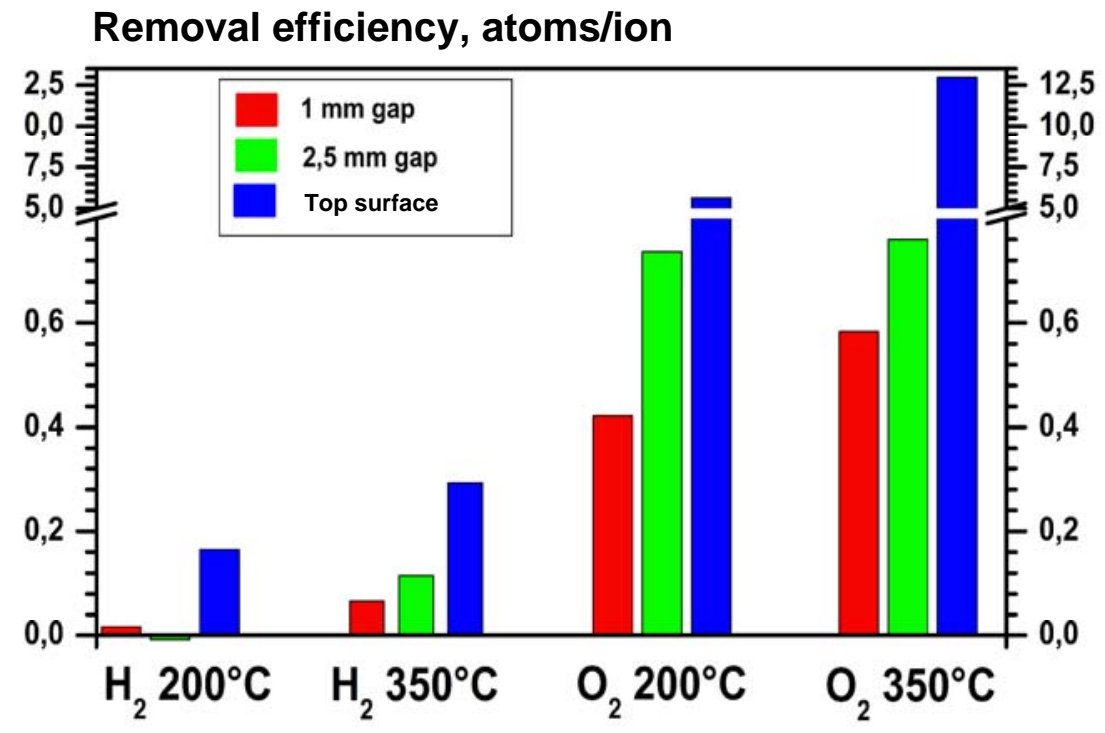

Figure 4. Comparison of deposit removal efficiency, normalized to the impinging plasma flux at the plasma-wetted top surface of the castellation. The data shown for various discharges in oxygen and hydrogen and for various temperatures of the castellation during the cleaning. 